\title{
Anhang 2d: Retweet-Netzwerk (Alternative und professionelle Nachrichtenmedien)
}

\begin{tabular}{|c|c|c|c|c|c|c|c|}
\hline Account & Modularity Class & $\begin{array}{c}\text { In- } \\
\text { degree }\end{array}$ & $\begin{array}{l}\text { Out- } \\
\text { degree }\end{array}$ & $\begin{array}{c}\text { De- } \\
\text { gree }\end{array}$ & $\begin{array}{l}\text { Closeness } \\
\text { Centrality }\end{array}$ & $\begin{array}{l}\text { Betweenness } \\
\text { Centrality }\end{array}$ & $\begin{array}{l}\text { Eigen. } \\
\text { Centr. }\end{array}$ \\
\hline SPIEGELONLINE & $\begin{array}{l}\text { Deutscher Mainstream, } \\
\text { Alternativmedien Typ II, IV }\end{array}$ & 2908 & 10 & 2918 & 0,120 & 21444292,795 & 0,310 \\
\hline Tagesspiegel & $\begin{array}{l}\text { Deutscher Mainstream, } \\
\text { Alternativmedien Typ II, IV }\end{array}$ & 1385 & 45 & 1430 & 0,202 & 96637865,327 & 0,153 \\
\hline SZ & $\begin{array}{l}\text { Deutscher Mainstream, } \\
\text { Alternativmedien Typ II, IV }\end{array}$ & 1336 & 3 & 1339 & 0,124 & 10675069,397 & 0,144 \\
\hline zeitonline & $\begin{array}{l}\text { Deutscher Mainstream, } \\
\text { Alternativmedien Typ II, IV }\end{array}$ & 1117 & 11 & 1128 & 0,107 & 6839027,224 & 0,118 \\
\hline berlindirekt & $\begin{array}{l}\text { Deutscher Mainstream, } \\
\text { Alternativmedien Typ II, IV }\end{array}$ & 1064 & 1 & 1065 & 1,000 & 70642,355 & 0,115 \\
\hline taz_news & $\begin{array}{l}\text { Deutscher Mainstream, } \\
\text { Alternativmedien Typ II, IV }\end{array}$ & 688 & 0 & 688 & 0,000 & 0,000 & 0,075 \\
\hline FridayForFuture & $\begin{array}{l}\text { Deutscher Mainstream, } \\
\text { Alternativmedien Typ II, IV }\end{array}$ & 548 & 0 & 548 & 0,000 & 0,000 & 0,058 \\
\hline politicalbeauty & $\begin{array}{l}\text { Deutscher Mainstream, } \\
\text { Alternativmedien Typ II, IV }\end{array}$ & 500 & 0 & 500 & 0,000 & 0,000 & 0,053 \\
\hline telepolis_news & $\begin{array}{l}\text { Deutscher Mainstream, } \\
\text { Alternativmedien Typ II, IV }\end{array}$ & 496 & 2 & 498 & 0,563 & 120647,767 & 0,055 \\
\hline dpa & $\begin{array}{l}\text { Deutscher Mainstream, } \\
\text { Alternativmedien Typ II, IV }\end{array}$ & 461 & 13 & 474 & 1,000 & 885760,091 & 0,059 \\
\hline ZDFheute & $\begin{array}{l}\text { Deutscher Mainstream, } \\
\text { Alternativmedien Typ II, IV }\end{array}$ & 425 & 5 & 430 & 0,857 & 243629,973 & 0,046 \\
\hline BR24 & $\begin{array}{l}\text { Deutscher Mainstream, } \\
\text { Alternativmedien Typ II, IV }\end{array}$ & 406 & 20 & 426 & 0,180 & 101623186,328 & 0,047 \\
\hline WDR & $\begin{array}{l}\text { Deutscher Mainstream, } \\
\text { Alternativmedien Typ II, IV }\end{array}$ & 390 & 10 & 400 & 0,153 & 3414332,839 & 0,043 \\
\hline tagesschau_eil & $\begin{array}{l}\text { Deutscher Mainstream, } \\
\text { Alternativmedien Typ II, IV }\end{array}$ & 339 & 0 & 339 & 0,000 & 0,000 & 0,047 \\
\hline TeamKenFM & $\begin{array}{l}\text { Deutscher Mainstream, } \\
\text { Alternativmedien Typ II, IV }\end{array}$ & 323 & 2 & 325 & 1,000 & 1793,090 & 0,035 \\
\hline
\end{tabular}




\begin{tabular}{|c|c|c|c|c|c|c|c|}
\hline Account & Modularity Class & $\begin{array}{c}\text { In- } \\
\text { degree }\end{array}$ & $\begin{array}{l}\text { Out- } \\
\text { degree }\end{array}$ & $\begin{array}{l}\text { De- } \\
\text { gree }\end{array}$ & $\begin{array}{l}\text { Closeness } \\
\text { Centrality }\end{array}$ & $\begin{array}{l}\text { Betweenness } \\
\text { Centrality }\end{array}$ & $\begin{array}{l}\text { Eigen. } \\
\text { Centr. }\end{array}$ \\
\hline WAZ_Redaktion & $\begin{array}{l}\text { Deutscher Mainstream, } \\
\text { Alternativmedien Typ II, IV }\end{array}$ & 302 & 1 & 303 & 0,417 & 193494,578 & 0,032 \\
\hline MDR_SN & $\begin{array}{l}\text { Deutscher Mainstream, } \\
\text { Alternativmedien Typ II, IV }\end{array}$ & 247 & 5 & 252 & 0,105 & 69360199,357 & 0,027 \\
\hline NachDenkSeiten & $\begin{array}{l}\text { Deutscher Mainstream, } \\
\text { Alternativmedien Typ II, IV }\end{array}$ & 243 & 0 & 243 & 0,000 & 0,000 & 0,027 \\
\hline Volksverpetzer & $\begin{array}{l}\text { Deutscher Mainstream, } \\
\text { Alternativmedien Typ II, IV }\end{array}$ & 168 & 5 & 173 & 0,175 & 1792721,009 & 0,018 \\
\hline DanieleGanser & $\begin{array}{l}\text { Deutscher Mainstream, } \\
\text { Alternativmedien Typ II, IV }\end{array}$ & 148 & 0 & 148 & 0,000 & 0,000 & 0,016 \\
\hline SZ_TopNews & $\begin{array}{l}\text { Deutscher Mainstream, } \\
\text { Alternativmedien Typ II, IV }\end{array}$ & 139 & 0 & 139 & 0,000 & 0,000 & 0,015 \\
\hline SPIEGEL_Top & $\begin{array}{l}\text { Deutscher Mainstream, } \\
\text { Alternativmedien Typ II, IV }\end{array}$ & 133 & 0 & 133 & 0,000 & 0,000 & 0,015 \\
\hline lobbycontrol & $\begin{array}{l}\text { Deutscher Mainstream, } \\
\text { Alternativmedien Typ II, IV }\end{array}$ & 131 & 0 & 131 & 0,000 & 0,000 & 0,014 \\
\hline tazgezwitscher & $\begin{array}{l}\text { Deutscher Mainstream, } \\
\text { Alternativmedien Typ II, IV }\end{array}$ & 131 & 6 & 137 & 0,100 & 808553,417 & 0,014 \\
\hline BILD & $\begin{array}{l}\text { Deutscher Mainstream, } \\
\text { Boulevard }\end{array}$ & 4351 & 2 & 4353 & 0,135 & 31943379,956 & 0,481 \\
\hline DerSPIEGEL & $\begin{array}{l}\text { Deutscher Mainstream, } \\
\text { Boulevard }\end{array}$ & 1366 & 2 & 1368 & 1,000 & 90918,633 & 0,150 \\
\hline focusonline & $\begin{array}{l}\text { Deutscher Mainstream, } \\
\text { Boulevard }\end{array}$ & 1010 & 5 & 1015 & 0,667 & 218007,783 & 0,120 \\
\hline morgenpost & $\begin{array}{l}\text { Deutscher Mainstream, } \\
\text { Boulevard }\end{array}$ & 1003 & 0 & 1003 & 0,000 & 0,000 & 0,117 \\
\hline FOCUS_TopNews & $\begin{array}{l}\text { Deutscher Mainstream, } \\
\text { Boulevard }\end{array}$ & 436 & 4 & 440 & 0,471 & 18905,517 & 0,051 \\
\hline focuspanorama & $\begin{array}{l}\text { Deutscher Mainstream, } \\
\text { Boulevard }\end{array}$ & 253 & 4 & 257 & 0,471 & 1811,097 & 0,030 \\
\hline BILD_Berlin & $\begin{array}{l}\text { Deutscher Mainstream, } \\
\text { Boulevard }\end{array}$ & 73 & 0 & 73 & 0,000 & 0,000 & 0,009 \\
\hline Tag24B & $\begin{array}{l}\text { Deutscher Mainstream, } \\
\text { Boulevard }\end{array}$ & 64 & 0 & 64 & 0,000 & 0,000 & 0,008 \\
\hline BILD_Ruhrgebiet & $\begin{array}{l}\text { Deutscher Mainstream, } \\
\text { Boulevard }\end{array}$ & 53 & 5 & 58 & 0,119 & 1412728,156 & 0,006 \\
\hline BILD_Promis & $\begin{array}{l}\text { Deutscher Mainstream, } \\
\text { Boulevard }\end{array}$ & 52 & 0 & 52 & 0,000 & 0,000 & 0,005 \\
\hline BILD_Koeln & $\begin{array}{l}\text { Deutscher Mainstream, } \\
\text { Boulevard }\end{array}$ & 30 & 0 & 30 & 0,000 & 0,000 & 0,003 \\
\hline RolandTichy & $\begin{array}{l}\text { Alternativmedien Typ I, } \\
\text { Boulevard }\end{array}$ & 3300 & 17 & 3317 & 0,195 & 25257146,35 & 0,386 \\
\hline
\end{tabular}




\begin{tabular}{|c|c|c|c|c|c|c|c|}
\hline Account & Modularity Class & $\begin{array}{c}\text { In- } \\
\text { degree }\end{array}$ & $\begin{array}{c}\text { Out- } \\
\text { degree }\end{array}$ & $\begin{array}{l}\text { De- } \\
\text { gree }\end{array}$ & $\begin{array}{l}\text { Closeness } \\
\text { Centrality }\end{array}$ & $\begin{array}{c}\text { Betweenness } \\
\text { Centrality }\end{array}$ & $\begin{array}{l}\text { Eigen. } \\
\text { Centr. }\end{array}$ \\
\hline HGMaassen & $\begin{array}{l}\text { Alternativmedien Typ I, } \\
\text { Boulevard }\end{array}$ & 2285 & 4 & 2289 & 0,153 & 5602741,256 & 0,274 \\
\hline Hartes_Geld & $\begin{array}{l}\text { Alternativmedien Typ I, } \\
\text { Boulevard }\end{array}$ & 1993 & 0 & 1993 & 0,000 & 0,000 & 0,236 \\
\hline TichysEinblick & $\begin{array}{l}\text { Alternativmedien Typ I, } \\
\text { Boulevard }\end{array}$ & 1818 & 9 & 1827 & 0,168 & 3080001,806 & 0,219 \\
\hline burger_ein & $\begin{array}{l}\text { Alternativmedien Typ I, } \\
\text { Boulevard }\end{array}$ & 1733 & 35 & 1768 & 0,225 & 8161719,846 & 0,220 \\
\hline SteinbachErika & $\begin{array}{l}\text { Alternativmedien Typ I, } \\
\text { Boulevard }\end{array}$ & 1493 & 6 & 1499 & 0,195 & 3602485,683 & 0,177 \\
\hline jouwatch & $\begin{array}{l}\text { Alternativmedien Typ I, } \\
\text { Boulevard }\end{array}$ & 1150 & 0 & 1150 & 0,000 & 0,000 & 0,146 \\
\hline KoeppelRoger & $\begin{array}{l}\text { Alternativmedien Typ I, } \\
\text { Boulevard }\end{array}$ & 1082 & 0 & 1082 & 0,000 & 0,000 & 0,133 \\
\hline deutsch365 & $\begin{array}{l}\text { Alternativmedien Typ I, } \\
\text { Boulevard }\end{array}$ & 1061 & 1 & 1062 & 1,000 & 11828,442 & 0,129 \\
\hline Junge_Freiheit & $\begin{array}{l}\text { Alternativmedien Typ I, } \\
\text { Boulevard }\end{array}$ & 924 & 0 & 924 & 0,000 & 0,000 & 0,108 \\
\hline AfD_Support & $\begin{array}{l}\text { Alternativmedien Typ I, } \\
\text { Boulevard }\end{array}$ & 904 & 8 & 912 & 0,196 & 1600288,650 & 0,111 \\
\hline bazonline & $\begin{array}{l}\text { Alternativmedien Typ I, } \\
\text { Boulevard }\end{array}$ & 874 & 1 & 875 & 1,000 & 72417,600 & 0,102 \\
\hline BILD_Politik & $\begin{array}{l}\text { Alternativmedien Typ I, } \\
\text { Boulevard }\end{array}$ & 709 & 2 & 711 & 0,152 & 2302854,112 & 0,090 \\
\hline krone_at & $\begin{array}{l}\text { Alternativmedien Typ I, } \\
\text { Boulevard }\end{array}$ & 700 & 6 & 706 & 0,146 & 4212856,117 & 0,087 \\
\hline Einzelfallinfos & $\begin{array}{l}\text { Alternativmedien Typ I, } \\
\text { Boulevard }\end{array}$ & 613 & 0 & 613 & 0,000 & 0,000 & 0,078 \\
\hline wach_jetzt_auf & $\begin{array}{l}\text { Alternativmedien Typ I, } \\
\text { Boulevard }\end{array}$ & 609 & 13 & 622 & 0,207 & 658313,031 & 0,080 \\
\hline Zeitgeschehen_ & $\begin{array}{l}\text { Alternativmedien Typ I, } \\
\text { Boulevard }\end{array}$ & 455 & 103 & 558 & 0,240 & 8925351,494 & 0,058 \\
\hline unzensuriert & $\begin{array}{l}\text { Alternativmedien Typ I, } \\
\text { Boulevard }\end{array}$ & 431 & 0 & 431 & 0,000 & 0,000 & 0,056 \\
\hline $\begin{array}{l}\text { Pressecop- } \\
24 \text { com }\end{array}$ & $\begin{array}{l}\text { Alternativmedien Typ I, } \\
\text { Boulevard }\end{array}$ & 343 & 3 & 346 & 1,000 & 30167,446 & 0,048 \\
\hline srfnews & Schweiz-Community & 1985 & 14 & 1999 & 0,136 & 17088648,504 & 0,215 \\
\hline tagesanzeiger & Schweiz-Community & 541 & 33 & 574 & 0,156 & 11255311,943 & 0,060 \\
\hline NZZaS & Schweiz-Community & 496 & 36 & 532 & 0,170 & 13019176,040 & 0,064 \\
\hline Knutti_ETH & Schweiz-Community & 482 & 17 & 499 & 0,150 & 5680880,087 & 0,052 \\
\hline Blickch & Schweiz-Community & 380 & 10 & 390 & 0,144 & 3084969,849 & 0,045 \\
\hline watson_news & Schweiz-Community & 295 & 19 & 314 & 0,149 & 2876820,942 & 0,032 \\
\hline
\end{tabular}




\begin{tabular}{|c|c|c|c|c|c|c|c|}
\hline Account & Modularity Class & $\begin{array}{c}\text { In- } \\
\text { degree }\end{array}$ & $\begin{array}{c}\text { Out- } \\
\text { degree }\end{array}$ & $\begin{array}{l}\text { De- } \\
\text { gree }\end{array}$ & $\begin{array}{l}\text { Closeness } \\
\text { Centrality }\end{array}$ & $\begin{array}{l}\text { Betweenness } \\
\text { Centrality }\end{array}$ & $\begin{array}{l}\text { Eigen. } \\
\text { Centr. }\end{array}$ \\
\hline Wochenzeitung & Schweiz-Community & 216 & 13 & 229 & 0,165 & 5675027,775 & 0,024 \\
\hline JolandaSpiess & Schweiz-Community & 206 & 30 & 236 & 0,194 & 17309279,115 & 0,022 \\
\hline persoenlichcom & Schweiz-Community & 179 & 8 & 187 & 0,165 & 7076688,587 & 0,022 \\
\hline srfsport & Schweiz-Community & 173 & 5 & 178 & 0,127 & 2957669,387 & 0,020 \\
\hline NZZzuerich & Schweiz-Community & 165 & 7 & 172 & 0,161 & 2054573,757 & 0,026 \\
\hline SVPch & Schweiz-Community & 156 & 0 & 156 & 0,000 & 0,000 & 0,018 \\
\hline NZZWissen & Schweiz-Community & 139 & 4 & 143 & 0,160 & 1150518,260 & 0,02 \\
\hline derbund & Schweiz-Community & 123 & 9 & 132 & 0,144 & 1627887,538 & 0,014 \\
\hline FDP_Liberalen & Schweiz-Community & 119 & 14 & 133 & 0,165 & 3174457,568 & 0,013 \\
\hline NZZAusland & Schweiz-Community & 107 & 17 & 124 & 0,164 & 399017,595 & 0,019 \\
\hline kathch & Schweiz-Community & 105 & 14 & 119 & 0,160 & 4526888,033 & 0,012 \\
\hline nzzwirtschaft & Schweiz-Community & 102 & 47 & 149 & 0,169 & 3698278,680 & 0,018 \\
\hline NZZSport & Schweiz-Community & 98 & 9 & 107 & 0,160 & 800984,952 & 0,017 \\
\hline srfbasel & Schweiz-Community & 95 & 1 & 96 & 0,000 & 0,000 & 0,013 \\
\hline adfichter & Schweiz-Community & 92 & 11 & 103 & 0,168 & 3988477,760 & 0,010 \\
\hline BernerZeitung & Schweiz-Community & 91 & 14 & 105 & 0,126 & 2036832,335 & 0,010 \\
\hline grunliberale & Schweiz-Community & 91 & 15 & 106 & 0,164 & 1842457,393 & 0,010 \\
\hline $\begin{array}{l}\text { RepublikMa- } \\
\text { gazin }\end{array}$ & Schweiz-Community & 86 & 32 & 118 & 0,163 & 4429130,342 & 0,010 \\
\hline Weltwoche & Schweiz-Community & 66 & 1 & 67 & 0,000 & 0,000 & 0,009 \\
\hline ZwitscherLAMM & Schweiz-Community & 3 & 1 & 4 & 0,101 & 2287,965 & 0,000 \\
\hline derStandardat & Österreich-Community & 1472 & 5 & 1477 & 0,144 & 190304366,950 & 0,160 \\
\hline ArminWolf & Österreich-Community & 756 & 25 & 781 & 0,169 & 37115920,454 & 0,084 \\
\hline florianklenk & Österreich-Community & 381 & 11 & 392 & 0,161 & 3727482,687 & 0,04 \\
\hline DiePressecom & Österreich-Community & 366 & 0 & 366 & 0,000 & 0,000 & 0,04 \\
\hline futurezoneat & Österreich-Community & 270 & 43 & 313 & 0,199 & 22033856,334 & 0,029 \\
\hline Natascha_Strobl & Österreich-Community & 255 & 1 & 256 & 0,150 & 1732518,055 & 0,027 \\
\hline webstandardat & Österreich-Community & 247 & 1 & 248 & 1,000 & 22608,957 & 0,027 \\
\hline misik & Österreich-Community & 234 & 7 & 241 & 0,164 & 1188396,919 & 0,025 \\
\hline FAZ_NET & Österreich-Community & 195 & 0 & 195 & 0,000 & 0,000 & 0,023 \\
\hline KURIERat & Österreich-Community & 180 & 4 & 184 & 0,131 & 1342564,659 & 0,020 \\
\hline PolitikStandard & Österreich-Community & 162 & 0 & 162 & 0,000 & 0,000 & 0,018 \\
\hline profilonline & Österreich-Community & 147 & 17 & 164 & 0,163 & 2881397,410 & 0,018 \\
\hline WienerZeitung & Österreich-Community & 109 & 3 & 112 & 0,117 & 507175,911 & 0,012 \\
\hline sn_aktuell & Österreich-Community & 105 & 0 & 105 & 0,000 & 0,000 & 0,012 \\
\hline Kontrast_at & Österreich-Community & 90 & 0 & 90 & 0,000 & 0,000 & 0,010 \\
\hline
\end{tabular}




\begin{tabular}{llrrrrrr}
\hline \multicolumn{1}{c}{ Account } & Modularity Class & $\begin{array}{c}\text { In- } \\
\text { degree }\end{array}$ & $\begin{array}{r}\text { Out- } \\
\text { degree }\end{array}$ & $\begin{array}{r}\text { De- } \\
\text { gree }\end{array}$ & $\begin{array}{c}\text { Closeness } \\
\text { Centrality }\end{array}$ & $\begin{array}{c}\text { Betweenness } \\
\text { Centrality }\end{array}$ & $\begin{array}{r}\text { Eigen. } \\
\text { Centr. }\end{array}$ \\
\hline SPOE_at & Österreich-Community & 76 & 10 & 86 & 0,146 & 560480,246 & 0,008 \\
\hline daswasfehlt & Österreich-Community & 58 & 4 & 62 & 0,170 & 550822,301 & 0,006 \\
\hline NZZ & Internationale Community & 5650 & 132 & 5782 & 0,190 & 171615955,497 & 0,630 \\
\hline sternde & Internationale Community & 3208 & 8 & 3216 & 0,121 & 41801334,092 & 0,336 \\
\hline KRLS & Internationale Community & 2586 & 0 & 2586 & 0,000 & 0,000 & 0,262 \\
\hline Foreign_Cat & Internationale Community & 546 & 9 & 555 & 0,162 & 8539639,278 & 0,056 \\
\hline
\end{tabular}




\section{Medienwissenschaft}
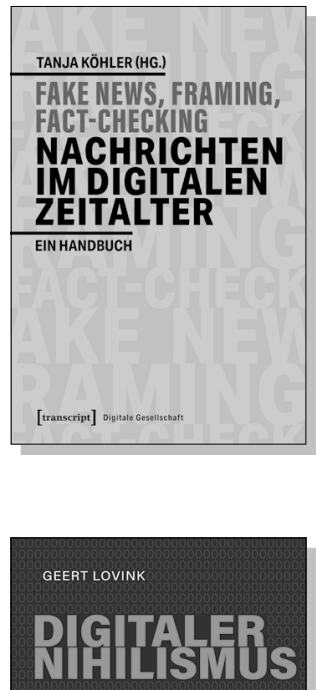

THESEN ZUR DUNKLEN SEITE DER PLATTFORMEN

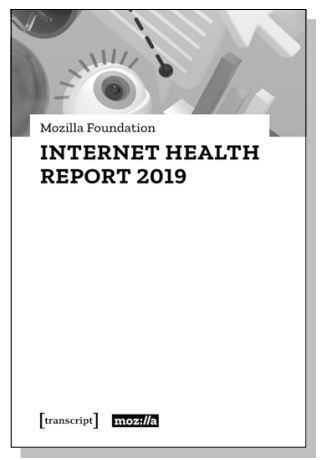

Tanja Köhler (Hg.)

Fake News, Framing, Fact-Checking: Nachrichten im digitalen Zeitalter Ein Handbuch

2020, 568 S., kart., 41 SW-Abbildungen 39,00€ (DE), 978-3-8376-5025-9

E-Book:

PDF: 38,99 € (DE), ISBN 978-3-8394-5025-3

Geert Lovink

\section{Digitaler Nihilismus}

Thesen zur dunklen Seite der Plattformen

2019, 242 S., kart.

24,99€ (DE), 978-3-8376-4975-8

E-Book:

PDF: $21,99 €$ (DE), ISBN 978-3-8394-4975-2

EPUB: $21,99 €$ (DE), ISBN 978-3-7328-4975-8

Mozilla Foundation

Internet Health Report 2019

2019, 118 p., pb., ill.

19,99€ (DE), 978-3-8376-4946-8

E-Book: available as free open access publication

PDF: ISBN 978-3-8394-4946-2 


\section{Medienwissenschaft}

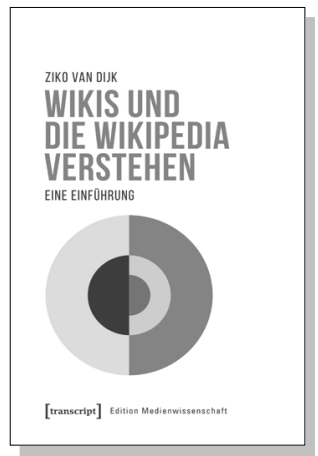

Ziko van Dijk

Wikis und die Wikipedia verstehen

Eine Einführung

März 2021, 340 S., kart.,

Dispersionsbindung, 13 SW-Abbildungen

35,00€ (DE), 978-3-8376-5645-9

E-Book: kostenlos erhältlich als Open-Access-Publikation PDF: ISBN 978-3-8394-5645-3

ISBN 978-3-7328-5645-9

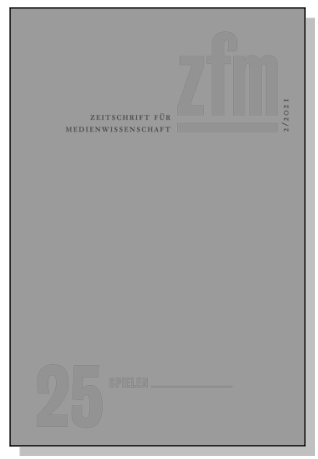

Gesellschaft für Medienwissenschaft (Hg.)

Zeitschrift für Medienwissenschaft

Jg. 13, Heft 2/2021: Spielen

September 2021, 180 S., kart.

24,99 € (DE), 978-3-8376-5400-4

E-Book: kostenlos erhältlich als Open-Access-Publikation

PDF: ISBN 978-3-8394-5400-8

ISBN 978-3-7328-5400-4

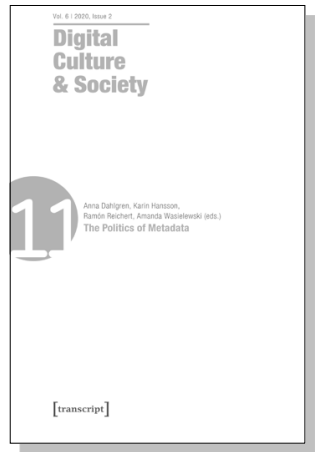

Anna Dahlgren, Karin Hansson,

Ramón Reichert, Amanda Wasielewski (eds.)

Digital Culture \& Society (DCS)

Vol. 6, Issue 2/2020 - The Politics of Metadata

June 2021, 274 p., pb., ill.

29,99€ (DE), 978-3-8376-4956-7

E-Book:

PDF: $29,99 €$ (DE), ISBN 978-3-8394-4956-1 
